\title{
THE EFFECT OF HEALTH EDUCATION IN THE MANAGEMENT OF TYPE 2 DIABE'TES AND 'THE SRI LANKAN PERSPECTIVE: A REVIEW
}

\section{Cooray BPR ${ }^{1}$, Ball $\mathbf{P A}^{2}$, Morrissey $\mathbf{H}^{3}$, Waidyarathne $\mathrm{EI}^{4}$.}

${ }^{1}$ School of Psychological and Clinical Sciences, Charles Darwin University, Australia, ${ }^{2}$ School of Pharmacy, Faculty of Science and Engineering, University of Wolverhampton, United Kingdom, ${ }^{3}$ School of Pharmacy, Faculty of Science and Engineering, University of Wolverhampton, United Kingdom, ${ }^{4}$ Faculty of Medicine, University of Ruhuna, Sri Lanka.

\section{ABSTRACT}

A high prevalence of diabetes and its microvascular and macrovascular complications constitute a significant burden on healthcare systems. Self-management through patient education contributes to reduce the burden of uncontrolled diabetes. Although there are a number of proven strategies in providing diabetes care education, there is insufficient evidence to recommend a specific type of education for our population. The aim of this article is to critically review published studies on Type 2 diabetes health literacy to establish a culturally adapted, structured education program on glycemic control for the Sri Lankans.

The review was limited to journal articles published in PubMed, Google Scholar and Google databases. Article search was done using the search headings of "patient education, type 2 diabetes and Sri Lankan studies". All the articles found in Sri Lankan journals and other important international articles on the topic during the last decade were also reviewed.

Poor health literacy is one of the major barriers that has limited access to healthcare facilities and reduce health outcomes. Although recent research has focused on health literacy as an intervention in the treatment of diabetes, none of the Sri Lankan studies have measured its effect on glycemic control in type 2 diabetes. This review has raised the need for measuring the impact of health education on management of type 2 diabetes. This could be adopted to develop targeted interventions on patient education to be implemented in glycemic control of Type 2 diabetes in Sri Lanka.

\section{INTRODUCTION}

The occurrence of diabetes shows substantial inconsistencies across the socioeconomic quintiles and is different, for different stages of life (1). This has become a major problem in low and middle socioeconomic countries where it has a significant influence on productivity and the overall economic output that leads to a considerable burden on health care and welfare systems (2).

Type 2 diabetes mellitus (T2DM) is the commonest type of diabetes, occurring as a heterogeneous disorder that affects the young, middle-aged and elderly. This metabolic disorder usually encompasses dyslipidemia, high blood pressure, and central obesity, reflecting the heterogeneity of different phenotypes (3).

Sri Lanka is amongst the countries with the highest diabetes prevalence rates in the world. As stated in an International Federation of Diabetes Atlas, 1.1 million cases of all types of diabetes were recorded in Sri Lanka in 2014 and the majority of them were suffering from T2DM. It has also revealed that there are a substantial number of pre-diabetics who were not included in these statistics (4). The high prevalence of diabetes and the associated microvascular and macrovascular complications can have major socioeconomic consequences that negatively affect the individual, their family and the society (5). In order to reduce the burden on health- -care systems, control of diabetes has become one of the priority components of healthcare management.

It is a well-recognized fact that the level of understanding on self-management determines the outcome of a chronic disease. Similarly, considering the nature of the disease, the degree of selfawareness on diabetes and its management determine the level of control and overall outcome of the disease. Understanding the important role of self-management of diabetes improve the adherence to therapy including both medications and lifestyle ensure better outcome via the influence on patient behavior, knowledge and attitudes (6-8). There is ample evidence to suggest that health education and awareness programs have improved the 
overall outcome of these patients (811) and therefore, it has been incorporated as an integral part of diabetes management (12-14). With the recent research, it has been widely accepted that the major component of treatment success is achieved by the education and improving the selfmanagement skills of these patients $(10,11)$. It is well known that the presence of diabetes prolongs the hospital stay of patients. A study conducted in 2015 by Medagama et al. has confirmed this finding even in our clinical setup. It also highlights the fact that one-quarter of all diabetes-related admissions are for optimization of glucose control that could be easily achieved in the community with proper patient education (15) and shows the positive effect that could be brought about on hospital overcrowding. Two other recent studies conducted by Jayasuriya et al., 2015 and Amarasekara et al., 2015 demonstrated that the majority of our adults with T2DM did not achieve desired glycemic control, which highlights the failure of current diabetes care practices $(9,16)$.

There are many published studies on diabetes education and the benefit on the overall outcome of these patients. However, there was limited research carried in this area of diabetes describing the impact of health education on glycemic control among T2DM patients in Sri Lanka. The aim of this exercise was to review the literature on health literacy among patients with T2DM and to introduce a culturally adapted, structured, pharmacist-delivered education program on glycaemic control. This paper constitutes part of a current study, which is investigating the potential role of pharmacists in improving patient health literacy and effective self-management of their T2DM in Sri Lanka.

\section{METHOD}

A review of the literature was performed to identify studies that evaluated the effect of diabetes education on T2DM patient self-management and diabetes control, both globally and specific to Sri Lanka. The electronic databases used for this study were PubMed, Science Direct, Google Scholar and Google search engine. The search was limited to articles published in the English language (which includes all known Sri Lankan medical journals) and conducted in humans. The search used the following headings: Patient education, type 2 diabetes, T2DM and Sri Lankan diabetes studies. A cross reference search was conducted to include articles of T2DM studies published in other Sri Lankan journals that were not included in indexed databases through google search engine. In order to increase the number of articles included in the review, an additional search was made based on keywords glycated hemoglobin test, $\mathrm{HbA}_{1 \mathrm{C}}$, and fasting blood glucose level. The articles that were mainly focusing on gestational diabetes and type 1 diabetes were excluded.

\section{RESULTS}

\section{Definition of type 2 diabetes}

T2DM is defined as tissues resistance to the action of insulin combined with the deficiency in insulin secretion due to the progressive lowering of insulin secretion by the $\beta$ cells that are inadequate to overwhelm the resistance, leading to T2DM. This also affects fat metabolism resulting in increased free fatty acid flux, triglyceride levels and reciprocally low levels of high-density lipoprotein (HDL) with the increased risk of developing micro and macrovascular complications (17).

\section{Cardio-metabolic risk profile of Sri Lankan patients with type 2 diabetes}

A study on "Evaluation of common Type 2 Diabetes risk variants in a South Asian population of Sri Lankan descent" stated that most T2DM risk variants identified in Sri Lankans were comparable to those in Europeans (18). The identified modifiable risk factors were the obesity, physical inactivity, hypertension, increased amount of high-density lipo- -protein and /or triglycerides, history of gestational diabetes or delivery of a baby weighing more than 4 kilograms (91b), history of vascular disease and polycystic ovarian syndrome $(8,19-22)$. One of the major risk factors associated with T2DM was the obesity, which occurs due to abnormal or excessive fat accumulation in the body, which is also associated with dyslipidemia, hypertension, other cardiovascular diseases, non-alcoholic fatty liver disease and other conditions including renal impairment $(3,23)$. A recent study by Katulanda et al., 2015 revealed that, even though morbid obesity is commonly accompanied by diabetes, there is an inconsistency between obesity data derived on the international categorization of obesity and the obesity related co-morbidities based on the prevalence of diabetes and cardiovascular diseases (24). Physical inactivity is another risk factor that contributed to the total risk of developing T2DM. With the economic development and technical advances, the lifestyle of the people has become more sedentary especially in the urban population of Sri Lanka as indicated by Katulanda et al. in 2008 and has been the reason for the higher prevalence of T2DM in urban population (25). Furthermore, Ranasinghe, 2014 and Siyambalapitiya et al., 2012, found that adherence to regular exercise and physical activity were poor among T2DM patients in Sri Lanka $(26,27)$. Those who adhered to regular exercise were commonly the males than females and therefore had fewer disease complications. Another study provided similar evidence indicating the need for the introduction of new strategies to change the attitude towards the physical activity of patients, of both genders, to achieve their individual glycemic targets (2628).

\section{Complications of diabetes}

As a lifelong disease, T2DM requires a commitment to improve glycemic control in order to delay or diminish the onset and the progression of microvascular and macrovascular 
complications (5). The direct and indirect effects of T2DM on the vascular system are responsible for the morbidity and mortality in all types of Diabetes. The microvascular complications including retinopathy, nephropathy, and neuropathy are relatively specific to diabetes and occurrence of these complications (particularly the diabetic retinopathy) has been used to develop the diagnostic criteria for diabetes. Complication of diabetes in our population has been comparable with other countries of South East Asia (18).

\section{Monitoring of metabolic parameters}

Monitoring of the metabolic parameters, such as blood pressure, body weight, lipid profile, blood glucose, and glycated hemoglobin $\left(\mathrm{HbA}_{1} \mathrm{c}\right)$, is essential in the clinical management of patients with diabetes. Hypertension, obesity, and dyslipidemia are well-known risk factors for atherosclerosis and are common in T2DM patients and assist in the evaluation of treatment response of the patients (29). At the same time, chronic diseases increase the need for multiple medications associated with complex dosing and side-effects (30).

Over the last decade, the level of $\mathrm{HbA}_{1} \mathrm{c}$ is considered as the goldstandard measurement of the chronic glycemic condition. Glycated hemoglobin is formed when the normal hemoglobin A reacts nonenzymatically with glucose. This is a slow, concentration-dependent reaction proportional only to the concentration of $\mathrm{HbA}$ and glucose. Since $\mathrm{HbA}$ remains in the circulation for around 3 months, the amount of $\mathrm{HbA}_{1 \mathrm{C}}$ present is expressed as a percentage of $\mathrm{HbA}$, proportional to the glucose concentration over that time (31-33). As revealed by the UK Prospective Diabetes Study (UKPDS), it is being used as the most reliable means of gauging chronic glycemic control and has become the cornerstone for the assessment of diabetes and risk of complications (34). Thus, specific $\mathrm{HbA}_{1} \mathrm{c}$ targets for diabetes care were introduced with the goal of preventing or delaying the development of long-term complications. However, due to the unavailability of the $\mathrm{HbA}_{1} \mathrm{c}$ measurements in most of the government hospital in Sri Lanka, monitoring of glyacemic control in the majority of patients is still done with fasting and postprandial blood glucose measurements.

\section{Self-management of diabetes}

The WHO argues that improving selfmanagement of chronic conditions such as diabetes may have a big impact on individual health than any improvement due to specific medical treatments. Acceptance of having the disease and self-awareness of the disease and treatment are important components of self-management of their disease, which is guided by the person's judgment of their own ability to succeed in reaching the specific goal. Thus, improving such skills helps people to develop the confidence to manage their condition effectively. Hence, diabetes self-management incorporates behavioral, personal and environmental factors into daily performance of recommended activities. The recent studies on the impact of patient narratives on selfefficacy and self-care behaviors in T2DM have shown that a valuable component of T2DM selfmanagement programs could be achieved through specific goal-directed behavioral strategies such as 'action plan' or 'implementation plan'. This action plan would direct the patient to create their own strategies regarding when, where and how to perform specific goal-directed behaviors (3335). This also encourages the patients to play a more active and effective role in the management of their disease.

\section{Patient education of self-management of T2DM}

Education programs on selfmanagement are increasingly recognized not only as an essential component of chronic disease management, but also as part of secondary prevention and a means of reducing the burden of chronic illness on individuals and the community. The experience of drug interactions, polypharmacy, social stigma, and unawareness may be responsible for the poor compliance to therapy resulting in long-term complications of diabetes. Hence, improving knowledge, skills and self-management education can motivate a person to make positive lifestyle changes and sustain it, addressing their health beliefs concerns at the same time.

Epidemiologic data has shown that the prevalence of T2DM varies with ethnicity. Therefore, culturally competent behavioral interventions should be the focus of major national initiatives when carrying out research with the aim of developing a culturally appropriate outcome. Addressing translation issues for non-English speaking populations and exploring motivating factors and strategies for diabetes self-management process need to be focused (36).

Managing diabetes through patient education interventions requires a culturally modified, structured program targeted at changing the dietary habits, physical activity, and adherence to medications, cessation of smoking and reduction of alcohol consumption. The development of such a program is desirable with the consideration of the diversity of health literacy levels, ethnicity and socioeconomic background in developing countries (37-39).

As in many other developing countries, primary care for patients in Sri Lanka is provided by Diabetes and Endocrinology clinics in the government hospitals. Overcrowding of these clinics has limited the patient consultation to 2-4 minutes that mainly focuses on medication rather than on lifestyle changes $(15,38)$. Despite the limitations with the resources, patient education programs are carried out by the diabetes nurse educators in certain hospitals that have not reached all the patients with diabetes. This has created a gap between the proper diabetes education and the implementation of self-management of diabetes $(39,40)$. However, this problem could be easily overcome with the help of other health care professionals such as diabetes educators, nurses, and pharmacists on 
diabetes education. This was seen in the study "Diabetes Medication Assistance Service: The pharmacist's role in supporting patient selfmanagement of T2DM in Australia" (41).

Patient education is usually carried out as groups as well as a one-on-one basis. Some researchers emphasize that attending a group-based education can have better outcomes than one-on-one education, stating important opportunities to share and compare experiences, receive empathy and support, and revise negative selfconcepts (42). Other studies supported the effectiveness of one-on-one patient education methods for T2DM showing that health professionals imparting personalized knowledge and insight, to trigger the specific patient new behavior leading to better control of their disease prognosis, and in the case of the T2DM may contribute to better glycemic control than group education (40-43). When considering the limitations of resources, group education would be more practical and acceptable in Sri Lankan health setup. Although we have enough practical strategies and proven methods to improve self-management skills of patients, Sri Lanka is far behind in implementing these processes for our patients in clinical practice and it is high time that we take an initiative to find our own model to suit our patients.

\section{The impact of health literacy on diabetes management and its measurements}

WHO describes health literacy as "the cognitive and social skills and knowledge which determine the motivation and ability of individuals to gain access to, understand and use information and making critical health decisions to successfully manage their own health" (44). It has been instrumental in developing a therapeutic relationship and mutual understanding between the patient and the involved health practitioner and should be focused beyond information dissemination. It requires interaction, participation and critical analysis that lead to comprehensive understating of the health condition by improving the health literacy. Therefore, the achievements of the health education should be assessed not only through glycemic control but also through improvement in health literacy as well.

The health education impact questionnaire (heiQ ${ }^{\mathrm{TM}}$ ) is a tool that is used to obtain a comprehensive measurement of proximal outcomes of health education interventions. This was initiated in 2005 by Professor Richard Osborne and his colleagues of Deakin University, Australia; validated and undergone extensive psychometric testing across chronic conditions. It has the capacity to evaluate the individuals' ability to manage their chronic condition irrespective of the type of underlying disease. This is being applied to a broad range of chronic disease conditions with different settings, by the range of users, as it is already being validated in a variety of settings with different languages. $\mathrm{HeiQ}^{\mathrm{TM}}$ is consist of separate independent questions representing eight domains including; healthdirected activity; positive and active engagement in life; emotional distress; self-monitoring and insight; constructive attitudes and approaches; skill and technique acquisition; social integration and support and health service navigation. The items of heiQ ${ }^{\mathrm{TM}}$ were constructed with minimal ambiguity and colloquialisms, confirmed with people from low socioeconomic backgrounds and have the applicability to our clinical setup. However, it is not yet translated or adapted to Sri Lanka in such a way to assess the effect of health education after the delivery of one-on-one structured education program (45-47).

\section{DISCUSSION AND CONCLUSIONS}

Diabetes is one of the commonest non-communicable diseases with lots of long term complications and self management has been instrumental in preventing most of these complications. Recent studies in developed countries revealed the need of local research that is culturally appropriate and to establish primary care education for T2DM patients which can be used by any health professional including pharmacists that would improve patient awareness of responsibility towards their own health, in terms of self-management of disease progression, adherence to medication and lifestyle adjustments.

Compliance with Ethical Standards:

This study was funded by University Grant Commission, Sri Lanka (No: UGC/DRIC/PG/2015(i)/RUH/01) and no funding associated with this review article.

Ethical approval: This article does not contain any studies with human participants or animals performed by any of the authors.

Acknowledgements

Many thanks for Library staff of Charles Darwin University, Australia.

\section{REFERENCES}

1. World Health Organization, World Health statistics 2013, Geneva: World Health Organization Press. 2013. http://www.who.int/gho/publicatio ns/world health statistics/2013

2. Zhang X, Zhao X, Harris A. Chronic diseases and labor force participation in Australia. Journal of Health Economics. 2009; 28(1): 91-108.

3. Weerarathna TP. Individualizing treatment of type 2 diabetes. Sri Lanka Journal of Diabetes Endocrinology and Metabolism. 2014; 4:56-8.

4. International Diabetes Federation. International Diabetes Federation Diabetes Atlas, Brussels, Belgium: International Diabetes Federation. 2014.

http://www.idf.org/diabetesatlas.

5. Fowler MJ. Microvascular and Macrovascular Complications of Diabetes.Clinical Diabetes, 2008; 26(2): 77-82.

6. Tan MY, Magarey JM, Chee SS, Lee LF, Tan MH. A brief structured education programme enhances selfcare practices and improves glycemic control in Malaysians with poorly controlled diabetes. Health Education Research. 2011; 26(5): 896-907.

7. Campbell T, Dunt D, Fitzgerald JL, Gordon I. The impact of patient narratives on self-efficacy and selfcare in Australians with type 2 
diabetes: stage 1 results of a randomized trial. Health Promotion International 2015;30(3): 438-448.

8. Tang YH, Panq SM, Chan MF, Yeung GS, Yeung VT. Health literacy, complication awareness, and diabetic control in patients with type 2 diabetes mellitus. Journal of Advance Nursing. 2008; 62(1): 74-83.

9. Amarasekara AA, Fongkaew W, Wimalasekera SW, Turale S, Chanprasit, C. Cross-sectional study of glycemic control among adults with type 2 diabetes. Nursing and Health Sciences. 2015; 17(2): 223-8.

10. World Health Organization, Preparing a health care workforce for the 21st century : The challenge of Chronic condition, Geneva: World Health Organization Press. 2005. http:// mww.who.int/whr/2006/06 ch ap3 en.pdf

11. Skinner TC, Carey ME, Cradock S, Daly H, Davies MJ, Doherty Y et al. Diabetes Education and SelfManagement for Ongoing and Newly Diagnosed (DESMOND): process modelling of pilot study. Patient Education and Counselling. 2006; 64(13): 369-77.

12. Al-Qazaz HK, Sulaiman SA, Hassali MA, Shafie AA, Sundram S, Al-Nuri $\mathrm{R}$ et al. Diabetes knowledge, medication adherence and glycemic control among patients with type 2 diabetes. Internationl Journal of Clinical Pharmacology. 2011; 33(6): 1028-35.

13. Pandit AU, Tang JW, Bailey Sc, Dans TC, Bocchini MV, Persell SD et al. Education, literacy, and health: Mediating effects on hypertension knowledge and control. Patient Education and Counselling. 2009; 75(3): 381-5.

14. Norris SL, Lau J, Smith SJ, Schmid $\mathrm{CH}$, Engelgau MM. SelfManagement education for adults with Type 2 Diabetes. Diabetes Care. 2002; 25(7): 1159-71.

15. Medagama $\mathrm{AB}$, Bandara $\mathrm{R}$, Wijetunge R. The High Burden of In-Hospital Diabetes Mellitus at A Tertiary Care Hospital in Sri Lanka; A Case Control Study. Journal of Diabetes and Metabolism 2015; 06(03).

16. Jayasuriya R, Pinidiyapathirage $M G$, Jayawardena R, Kasturiratne A, De Soyza P, Godamunna $\mathrm{P}$ et al. Translational research for Diabetes Self-Management in Sri Lanka: A randomized controlled trial. Primary Care Diabetes. 2015; 9(5): 338-45.

17. World Health Organisation, Definition and diagnosis of diabetes mellitus and intermediate hyperglycemia. Geneva: World Health Organization Press. 2006. bttp:/ / www. who.int/diabetes/publications/diagno sis diabetes2006

18. Hassanali N, De Silva NMG, Rayner NW, Barret A, Benett AJ, Groves CJ et al. Evaluation of common type 2 diabetes risk variants in a South Asian population of Sri Lankan descent. PLoS One. 2014; 9(6): 98608.

19. Sarkar U, KarterAJ, Liu JL. Hypoglycemia is more common among Type 2 Diabetes patients with limited health literacy. Journal of General Internal Medicine. 2010; 25(9): 962-68.

20. Thomson GA, Medagama A, Dissanayake A, Lenora D, Kuarihamy W, Bushby P, et al. Pandemic diabetes: can developed-world health professionals do more to support care in developing countries? European Diabetes Nursing 2008; 5(1): 23-27.

21. Fernandez RF. Congruence between the Indian Diabetes Risk Score and Australian Type 2 Diabetes Risk Assessment tool screening in AsianIndians. Nurse Researcher. 2013;21(2): 36-9.

22. Bennett PN, Brown MJ. Endocrine system. Clinical Pharmacology ed. 9th ed: Churchill Livingstone; 2003.

23. Joseph TD, Talbert RT, Yee GC, Matzke. Pharmacotherapy- A Pathophysiologic Approach, 7th ed: Mac Grow Hill Medicine; 2008;1205-43.

24. Katulanda P, Jayawardena R. Use of ethnic specific BMI and waist cut-offs for prevention of diabetes and cardiovascular disease. Sri Lanka Journal of Diabetes, Endocrinology and Metabolism, 2015;5:1-3.

25. Katulanda P. Constantine GR, Mahesh JG, Sheriff $\mathrm{R}$, Seneviratne RD, Wijeratne $\mathrm{S}$ et al. Prevalence and projections of diabetes and pre-diabetes in adults in Sri Lanka-Sri Lanka Diabetes, Cardiovascular Study (SLDCS). Diabetic Medicine. 2008; 25(9): 1062-69.

26. Siyambalapitiya S, Gunathilake G, Perera I. Exercise: An essential component of diabetes management. Sri Lanka Journal of Diabetes Endocrinology and Metabolism. 2012; 2: 57-60.

27. Ranasinghe DC, Ranasinghe $P$, Jayawardena R, Mathews DR, Katulanda P. Evaluation of physical activity among adults with diabetes mellitus from Sri Lanka. International Archives of Medicine. 2014;7(1): 15.

28. Rankin D, Debbie D Cooke DD, Elliott J, Heller SR, Lawton J, et al. Supporting self-management after attending a structured education programme: a qualitative longitudinal investigation of type 1 diabetes patients' experiences and views. BMC Public Health 2012;12:652.
29. WHO expert consultation. Appropriate body-mass index for Asian populations and its implications for policy and intervention strategies. The Lancet. 2004; 363 (9403): 157-63.

30. Yuan C, Lai CWK, Chan LWC, Chow M, Law HKW, Ying M. The effect of diabetes self-management education on body weight, glycemic control, and other metabolic markers in patients with Type 2 Diabetes Mellitus. Journal of Diabetes Research. 2014; 761-89.

31. Russell CL, Ruppar TM, Matteson M. Improving medication adherence: moving from intention and motivation to a personal systems approach. Nursing Clinics of North America. 2011; 46(3): 271-81.

32. Somasundaram NP, Wijeyaratne $\mathrm{CN}$, De Silva S, Siribaddana S, Illangasekera U, Rajaratnam H. et al. Diabetes Mellitus: Glucose Control. Sri Lanka Journal of Diabetes Endocrinology and Metabolism. 2013; 3: 45-57.

33. Pathmanathan S, Somasundaram NP. HbA1C and diabetes - an overview, Sri L anka Journal of Diabetes, Endocrinology and Metabolism. 2013; 3: 104-107.

34. Group UK prostective Diabetes Study. Intensive blood-glucose control with sulphonylureas or insulin compared with conventional treatment and risk of complications in patients with type 2 diabetes (UKPDS 33). The Lancet. 1998; 352(9131): 83753.

35. Donald M, Dower J, Ware R, Mukandi B, Parekh S, Bain C Living with diabetes: Rationale, study design and baseline characteristics for an Australian prospective cohort study. BMC Public Health. 2012; 12: 8.

36. Jarvis J, Skinner TC, Carey ME, Davies MJ. How can structured selfmanagement patient education improve outcomes in people with type 2 diabetes? Diabetes Obesity and Metabolism 2010; 12(1): 9-12.

37. Speight J. Managing diabetes and preventing complications: what makes the difference? Medical Journal of Australia. 2013; 198(1): 16-7.

38. Baradaran HR, Knill-Jones RP, Wallia $\mathrm{S}$, Rodgers A. A controlled trial of the effectiveness of a diabetes education program in a multi-ethnic community in Glasgow. BMC Public Health. 2006; 6: 134 .

39. Sarkar U, Fisher L, Schillinger D. Is self-efficacy associated with diabetes self-management across race/ethnicity and health literacy? Diabetes Care. 2006; 29: 823-29.

40. Jayawardena M.H De S, Idampitiya C, Jayawarna C, Wanigasuriya $\mathrm{K}$, 
Thomson JA, Fernando DJS. An audit of standards of care at a Sri Lankan diabetic clinic. Diabetes Research and Clininical Practice. 2007; 75(2): 249-51.

41. Mitchell B, Armour C, Lee M, Song YJ, Stewart K, Peterson G et al. Diabetes Medication Assistance Service: the pharmacist's role in supporting patient self-management of type 2 diabetes (T2DM) in Australia. Patient Education and Counselling. 2011; 83(3): 288-94.

42. Lawton J, Rankin D. How do structured education programmes work? An ethnographic investigation of the dose adjustment for normal eating (DAFNE) programme for type 1 diabetes patients in the UK. Social Science and Medicine. 2010; 71(3): 48693.

43. Sandra Nolte, Elsworth GR, Osborne $\mathrm{RH}$. Absence of social desirability bias in the evaluation of chronic disease self-management interventions. Health and quality of life outcomes. 2013; 11(1): 114-22.

44. World Health Organisation, Health Promotion, Track 2-Health literacy and health behaviour. Geneva: World Health Organization Press. 2009. http://www.who.int/healthpromotio $\mathrm{n} /$ conferences $/ 7 \mathrm{gchp} / \operatorname{track} 2$

45. Epstein J, Osborne RH, Elsworth GR, Beaton DE, Guillemin F. Crosscultural adaptation of the Health Education Impact Questionnaire: experimental study showed expert committee, not back-translation, added value. Journal of Clinical Epidemiology. 2013;68(4)1-9.

46. Osborne RH, Batterham R, Livingston J. The evaluation of chronic disease self-management support across settings: the international experience of the health education impact questionnaire quality monitoring system. Nursing Clinics of North America. 2011;46(3): 255-70.

47. Morita R, Arakida M, Osborne RH, Nolte H, Elsworth GR, Mikami H. Adaptation and validation of the Japanese version of the Health Education Impact Questionnaire (heiQ-J) for the evaluation of selfmanagement education interventions. Japan Journal of Nursing Science. 2013; 10(2): 255-66. 